\title{
La Fleur des batailles Doolin de Maience publiée par Antoine Vérard (1501), Édition critique par Marie- Jane Pinvidic
}

\section{Maria Colombo Timelli}

\section{(2) OpenEdition \\ 1 Journals}

\section{Édition électronique}

URL : http://journals.openedition.org/studifrancesi/3679

DOI : 10.4000/studifrancesi.3679

ISSN : 2421-5856

Éditeur

Rosenberg \& Sellier

\section{Édition imprimée}

Date de publication : 1 décembre 2012

Pagination : 550-551

ISSN : 0039-2944

\section{Référence électronique}

Maria Colombo Timelli, «La Fleur des batailles Doolin de Maience publiée par Antoine Vérard (1501), Édition critique par Marie-Jane Pinvidic », Studi Francesi [En ligne], 168 (LVI | III) | 2012, mis en ligne le 30 novembre 2015, consulté le 07 mars 2021. URL : http://journals.openedition.org/studifrancesi/ 3679 ; DOI : https://doi.org/10.4000/studifrancesi.3679

Ce document a été généré automatiquement le 7 mars 2021.

\section{cc) $(9)$}

Studi Francesi è distribuita con Licenza Creative Commons Attribuzione - Non commerciale - Non opere derivate 4.0 Internazionale. 


\title{
La Fleur des batailles Doolin de Maience publiée par Antoine Vérard (1501), Édition critique par Marie-Jane Pinvidic
}

\author{
Maria Colombo Timelli
}

\section{RÉFÉRENCE}

La Fleur des batailles Doolin de Maience publiée par Antoine Vérard (1501), Édition critique par MARIE-JANE PINVIDIC, Paris, Honoré Champion, 2011 («Textes Littéraires de la Renaissance», 6), pp. 535.

1 C'est à Antoine Vérard que l'on doit l'editio princeps de La Fleur des batailles, Doolin de Maience, remaniement en prose de la chanson de geste Doon de Mayence: cette édition, datée dans le colophon mai 1501, est conservée dans au moins quatre exemplaires, dont un sur vélin (BnF, Rés. Vélins 624), enrichi de peintures, d'initiales et de pieds-demouche rehaussés en or, une de ces éditions somptueuses imitant la facture des manuscrits que le grand éditeur parisien réservait à des destinataires de la plus haute noblesse. Elle fut suivie par six autres au Xvi ${ }^{\mathrm{e}}$ siècle, à Paris et à Lyon, puis par d'autres encore, troyennes et rouennaises, jusqu'en 1626, preuve d'un succès durable. C'est assurément un texte qui méritait d'être édité selon des critères scientifiques et resitué dans le cadre des nouvelles recherches sur les réécritures en prose des $\mathrm{XV}^{\mathrm{e}}-\mathrm{XVI}^{\mathrm{e}}$ siècles.

Disons tout de suite que cette édition ne remplit pas ces conditions. L'Introduction déjà contient des ingénuités, sinon des fautes, qui prêteraient au sourire peut-être dans une thèse, pas dans un volume publié dans une collection sérieuse et renommée.

Dès les premières pages, consacrées à L'édition d'Antoine Vérard, c'est la confusion qui règne. On lit d'abord: «L'édition d'Antoine Vérard n'est pas la seule prose née de Doon de Maience»; alors qu'on apprendra, au début du paragraphe suivant, qu'«il n'existe, en 
fait, qu'une seule translation, celle de l'édition Vérard» (p.17, c'est moi qui souligne). Le même éditeur devient, à la page suivante, l'«auteur» de la mise en prose (p.18). Le deuxième chapitre, Antoine Vérard, sa vie et son œuvre, est fondé sur l'étude magistrale de Mary Beth Winn, mais les inadvertances sont nombreuses: p. 32 on fait allusion au «Maître Jacques de Besançon» (sic!); p. 34 La Fleur est définie comme «un roman chevaleresque» (le classement générique, nullement justifié, subit de nombreuses variations au sein même de l'Introduction). Encore: «Le prologue [...] n'a pas été conçu par Vérard, puisqu'il ne figure pas dans la liste établie par Mary Beth Winn» (pp.34-35); c'est sans doute l'inverse qu'il faudrait dire... Constatons par ailleurs que Mme Pinvidic parle modestement de son travail comme d'une 'réédition' (p. 23, puis de nouveau p. 64 et p. 71 ).

Le titre du chapitre 3, Les réimpressions de 'La Fleur', est surprenant, puisque les éditions des $\mathrm{XVI}^{\mathrm{e}}$ et $\mathrm{XVII}^{\mathrm{e}}$ siècles dont il sera question ne sont évidemment pas des «réimpressions». Là encore, il suffira de signaler quelques perles: «Trepperel, Lotrian, Janot utilisent pratiquement les mêmes bois gravés, et ces bois ressurgissent encore chez Bonfons...» (pp.38-39, c'est toujours moi qui souligne); «toutes les éditions reproduisent fidèlement le texte de Vérard, avec des transformations dues au rajeunissement de la langue. Seule, celle de Waesbergue [mais elle date de 1604] fait exception» (p. 39).

5 Je passe sur les fautes dans les citations (p. 40, colonne de droite: «accusee d'une d'une grande trahyson", par ailleurs on ne comprend pas pourquoi cette table compare le texte de Trepperel, et non pas celui de Vérard, à celui de Waesberge). Personnellement, je ne saurais comment interpréter la conclusion de ce même chapitre, p. 41: «Il semble donc que nous ayons deux proses imprimées bien différentes [il s'agirait des éditions du $\mathrm{XVI}^{\mathrm{e}}$ siècle d'une part, de celles du XVII ${ }^{\mathrm{e}}$ de l'autre], permettant un premier classement... Un autre classement, fondé sur les critères extérieurs, est aléatoire, et il parait plus opportun de parler de continuité; chaque édition marque un changement typographique, qui éclaire la tache [sic!] ingrate de la collation».

Suit le Résumé de 'La Fleur', devenue ici un «roman» (p. 43 et p. 47).

7 La discussion sur L'auteur et son modèle fait l'objet du chapitre 5: des trois manuscrits conservés qui ont transmis la chanson de geste, c'est celui de Montpellier qui semble offrir le texte le plus proche de la mise en prose (on corrigera, dans la citation à la p. 51, colonne de gauche «D'ont je sui» en «Dont je sui»).

8 Les surprises continuent dans l'analyse littéraire (Les particularités littéraires de 'La Fleur'): la référence la plus récente pour la pratique des mises en prose est le volume de Georges Doutrepont, incontournable, certes, mais comment ignorer la bibliographie de plus en plus riche des 30-40 dernières années? Voici alors que, «comme cet auteur [Doutrepont] le remarque à la suite de Léon Gautier, un texte mis en prose s'écarte toujours de l'original et ne peut jamais en être la copie absolue» (p. 53)! Et encore, p. 58: «le texte de La Fleur peut donner l'illusion de n'être qu'une copie [du modèle en vers]». $\mathrm{Ou}$, à propos de l'emploi du discours direct: «Le narrateur [...] mêle paroles rapportées et paroles proférées - la barre oblique, et non le point, souligne la continuité des discours», avec un beau mélange des rôles de l'auteur (du narrateur?) et de l'imprimeur (p. 62). Et, en conclusion: «Le romancier [à savoir l'auteur de la mise en prose] essaie de maintenir les caractéristiques du style épique [...], mais il ne parvient pas à une prose poétique» (p. 64). 
La langue de 'La Fleur' (chapitre 7, pp. 65-66) ne traite que de quelques graphies: de fait, l'analyse linguistique, très détaillée, se lit aux pp.325-378. Deux remarques: Mme Pinvidic, dont les connaissances de l'ancien français sont très approfondies, paraît moins à l'aise devant la variabilité du moyen français, et surtout tend à conserver à tout prix (on le verra pour ce qui est du traitement du texte) les formes de Vérard (mieux, de son imprimeur), dont pourtant est connu le peu de soin pour ces aspects. Quelques observations: plutôt que d'interpréter «le pluspart» (une seule occurrence) comme une forme dialectale du picard ou wallon (p. 349), j'aurais corrigé le texte; j'hésiterais à classer l'alternance graphique qui / quil(z) comme "confusion des formes», en y voyant le reflet de l'amuïssement de -l final (p. 356; même phénomène dans la graphie sil pour $s i$, un seul exemple, traité comme «enclise» p. 353). Une remarque aussi sur «l'ordre des mots dans la proposition» (traité aux pp. 376-377): Mme Pinvidic se limite à signaler «l'emploi de et en tête de proposition», sans nullement s'interroger justement sur le fait que le début de phrase dépend de la ponctuation adoptée (par l'imprimeur ou par l'éditeur critique?), en affirmant d'ailleur que «Et agit [...] comme le ferait un élément atone ou tonique» (sic, p. 377). L'étude linguistique se clôt sur une «Conclusion générale» de 6 lignes qui vaut la citation: «certains [traits linguistiques] appartiennent [...] aux régions du Nord et de l'Est et permettent de penser qu'à l'origine, la copie manuscrite de Doon de Maience, mise en prose par l'auteur de La Fleur, devait provenir des scripta de ces régions» (p. 378), bel exemple de confusion entre texte-source et mise en prose, manuscrit et scripta...

10 Le dernier chapitre de l'Introduction est consacré à l'Etablissement du texte: inutile de chercher, parmi les références, les conseils de Mario Roques ou les fascicules publiés par l'École des chartes. Le paragraphe sur la ponctuation s'ouvre par cette phrase: «De la ponctuation et du découpage du texte découle le système de références inspiré de livres tels que la Bible et le Coran» (p. 67: j'avoue ne pas comprendre). Les quelques remarques qui suivent («la barre oblique [...] peut avoir toutes les valeurs possibles», «la coupure d'un mot en fin de ligne [...] ne suit pas les règles du français moderne», ibidem) semblent révéler une très grande naïveté.

11 Le texte (pp. 85-257) reproduit l'exemplaire de l'édition Vérard conservé à Nantes, Musée Dobrée, Imp. 554: signalons que celui de la BnF, Rés. $Y^{2} .78$, est accessible dans Gallica. Là encore, de nombreuses remarques seraient possibles. Je me limite à signaler que le dévouement de Mme Pinvidic à son texte l'amène à conserver et parfois à défendre des leçons manifestement fautives (par ex. «car ce sont les mescreans», à lire «ce font», p. 108). D'autre part, son édition n'est pas exempte de fautes: p. 103, il faudra introduire des guillemets pour isoler le passage du discours rapporté au discours direct («la dame s'estoit ventee qu'elle auroit ung chevalier quil la deffenderoit contre deux 'et monstrera que nous, comme traistres, l'avons emprisonnee;' et jura Herchambault et la dame Susanne que...»). Encore: «son espee au poing, qui est grande et pensante» (p. 139); «quant il sera feru de nous d'eux» (p. 145).

12 Après le texte, on trouvera la reproduction des bois gravés (pp. 259-267), puis les «Notes du texte et variantes» (sic, pp. 269-323), très minutieuses; M.J. Pinvidic ne s'étant pas posé la question des rapports entre les imprimés, elle a néanmoins intégré parfois les leçons des manuscrits de la source en vers. On notera que l'«édition de J. Trepperel à Utrecht» (note 228, p. 269) désigne de fait l'exemplaire de cette édition conservé dans la bibliothèque de la ville néerlandaise (cf. p. 37). Inutile de renchérir sur ce qui aura déjà paru évident; une seule citation, tirée de la deuxième note au Prologue 
et concernant le syntagme «chevalier preux et hardy, filz du noble et chevalereux Guy»: «les mises en prose postérieures [comprendre: les imprimés postérieurs à Vérard] possédant le Prologue et la virgule...» (p. 270).

13 Là encore, nous ne partageons pas toujours les choix de l'éditrice, hésitant entre une prudence excessive et un interventionnisme non justifié. Un exemple: devant la leçon «en ces forestz a umbrees» (Vérard, 13v, à lire aumbrees, ce qui est confirmé par deux manuscrits du modèle en vers), Mme Pinvidic préfère corriger «ces forestz à umbre» uniquement parce que le $u$ de Vérard est celui du $u$ - initial, ce qu'elle explique longuement dans la note 17a-4, p. 285.

Le glossaire est très riche (pp. 379-497): il fournira certainement des informations utiles tant aux lecteurs de La Fleur qu'aux lexicographes; il est par conséquent ingrat de souligner quelques lacunes: l'acception érotique de mestier est bien attestée en moyen français («ilz firent leur desirer et se baiserent et accolerent ainsi qu'il affiert au mestier», p. 126, glosé comme 'activité, occupation' p. 449; on verra DMF 2010, ainsi que Rose Bidler, Dictionnaire érotique, Montréal, Ceres, 2002, s.v.); evangiles fém. («sur les sainctes evangiles de Dieu», p. 143, cf. DMF 2010) aurait mérité d'être relevé.

La Table des proverbes (pp. 499-507) suit l'ordre d'apparition dans le texte, ce qui n'est pas fait pour faciliter la consultation. La Table des noms propres (pp. 509-532) intègre beaucoup d'informations: elle comprend même Dieu, Jesu Crist, Marie (la Vierge)...; parmi les renseignements complémentaires, on trouvera, s.v. Petit Pont, que «la rue Saint Jacques se trouve dans le cinquième arrondissement de Paris»; M.J. Pinvidic, qui a introduit ce nom parce qu'il apparaît évidemment dans le colophon de Vérard, précise par ailleurs qu'il ne se lit pas dans Doon de Maience (p. 526).

Cette «réédition» nous provoque en somme un grand malaise, et nous fait exprimer le souhait que les éditions critiques des mises en prose, attendues parfois depuis longtemps et qui s'annoncent pour ces prochaines années, seront menées de façon plus scientifique. Sans aucun doute, La Fleur des batailles aurait mérité d'être mieux traitée. 